\title{
Spin-Peierls instability in a quantum spin chain with Dzyaloshinskii-Moriya interaction
}

\author{
O. Derzhko ${ }^{a *}$, J. Richter ${ }^{a \dagger}$, and O. Zaburannyi ${ }^{b}$ \\ ${ }^{a}$ Max-Planck-Institut für Physik komplexer Systeme, \\ Nöthnitzer Str. 38, 01187 Dresden, Germany \\ ${ }^{b}$ Institute for Condensed Matter Physics, \\ 1 Svientsitskii Str., L'viv-11, 79011, Ukraine
}

October 22, 2018

\begin{abstract}
We analysed the ground state energy of some dimerized spin- $\frac{1}{2}$ transverse $X X$ and Heisenberg chains with Dzyaloshinskii-Moriya (DM) interaction to study the influence of the latter interaction on the spinPeierls instability. We found that DM interaction may act either in favour of the dimerization or against it. The actual result depends on the dependence of DM interaction on the distortion amplitude in comparison with such dependence for the isotropic exchange interaction.
\end{abstract}

PACS numbers: 75.10.-b

Keywords: transverse $X X$ chain, Heisenberg chain, Dzyaloshinskii-Moriya interaction, spin-Peierls dimerization

Postal addresses:

Dr. Oleg Derzhko (corresponding author)

Oles' Zaburannyi

Institute for Condensed Matter Physics

1 Svientsitskii Street, L'viv-11, 79011, Ukraine

tel/fax: (0322) 761978

email: derzhko@icmp.lviv.ua

Prof. Johannes Richter

Institut für Theoretische Physik, Universität Magdeburg

P.O. Box 4120, D-39016 Magdeburg, Germany

tel: (0049) 3916718841

fax: (0049) 3916711217

email: Johannes.Richter@Physik.Uni-Magdeburg.DE

\footnotetext{
* On leave of absence from Institute for Condensed Matter Physics, 1 Svientsitskii Str., L'viv-11, 79011, Ukraine and Chair of Theoretical Physics, Ivan Franko National University in L'viv, 12 Drahomanov Str., L'viv-5, 79005, Ukraine

${ }^{\dagger}$ On leave of absence from Institut für Theoretische Physik, Universität Magdeburg, P.O. Box 4120, D-39016 Magdeburg, Germany
} 
The spin-Peierls instability is known as a magnetic analogue of the conventional Peierls instability in electronphonon systems. A uniform quantum spin chain at low temperatures may become unstable towards dimerization owing to the interaction with lattice degrees of freedom. This occurs because the dimerized lattice distortion lowers the magnetic energy by a greater amount than the increase in the elastic energy due to deformation. Starting in the 70s with organic compounds exhibiting spin-Peierls transition, the interest in the spin-Peierls instability of quantum spin chains was renewed with the discovery of the inorganic spin-Peierls compound $\mathrm{CuGeO}_{3}$ in 1993 [1, 2]. To model appropriately the spin degrees of freedom of the spin-Peierls compounds the pure Heisenberg chain as well as its modifications, which include frustration or interchain interaction, are considered. As a rule, since those models represent quantum many body systems, only approximate results can be obtained. However, some generic features of the spin-Peierls systems can be illustrated in a simplified but exactly solvable quantum spin model, namely, the transverse $X X$ chain [3, 4, 5, 6].

In the present paper we discuss the influence of the Dzyaloshinskii-Moriya (DM) interaction [7] on the spinPeierls dimerization in the adiabatic limit. The presence of $\mathrm{DM}$ interaction for $\mathrm{CuGeO}_{3}$ was proposed in Refs. [8, 9, 10] in order to explain the EPR and ESR experimental data. The structure of DM interaction in the cuprates was examined in Refs. [11, 12]. The influence of this interaction on the ground state properties of the one-dimensional and two-dimensional Heisenberg models was studied in Refs. 13, 14, 15. Besides, the multisublattice transverse $X X$ chain with DM interaction was introduced in Ref. [16], however, the spin-Peierls instability was discussed only in one limiting case (see below) in the absence of an external field. DM interaction was found to be present in a number of quasi-one-dimensional magnets (see, e.g., 17, 18] and also [19] in which $\mathrm{RbCoCl}_{3} \cdot 2 \mathrm{H}_{2} \mathrm{O}$ is described as a pure DM chain) and a study of one-dimensional DM Hamiltonians seems to be of great importance.

Several mechanisms which may destroy the dimerized phase in the Heisenberg chain were discussed in the literature, in particular, an external field or an Ising anisotropy. $X X$ anisotropy also suppresses the dimerized phase although does not destroy it completely. Therefore, initially one may expect that the appearance of an anisotropy in form of the DM interaction could act against dimerization. Although we shall find that the Heisenberg chain with increasing of DM interaction may become similar to the $X X$ chain both enhancing or suppressing of the dimerized phase are possible depending on the details of the distortion dependence of DM interaction.

In our study we follow the idea of Ref. [3] and compare the total ground state energy of the dimerized and uniform chains in the presence of DM interaction. First we demonstrate that in some cases the DM interaction can be eliminated by a spin coordinate transformation resulting in a model with an anisotropic exchange interaction. This observation permits us to study rigorously the influence of DM interaction on the spin-Peierls instability in the transverse $X X$ chain using the exact results for thermodynamic quantities of the regularly 
alternating transverse $X X$ chain obtained recently with the help of continued fractions [20]. (The approach exploiting continued fractions in contrast to the approaches used in previous works [3, 4, 5, 6, 16] allows one to consider in a similar way not only the dimerized lattice but also more complicated lattice distortions.) Further, we discuss the case of the Heisenberg chain with DM interaction using exact diagonalization of finite chains. The exact analytical findings for the $X X$ chain are helpful for the interpretation of the finite-chain results for more realistic Heisenberg chain. In the present study we are interested in describing the generic features originated by DM interaction and therefore no compound specific parameters are considered.

To begin with, we consider a nonuniform chain of $N \rightarrow \infty$ spins $\frac{1}{2}$ governed by the isotropic Heisenberg Hamiltonian with DM interaction

$$
H=\sum_{n} \Omega_{n} s_{n}^{z}+\sum_{n}\left(J_{n}\left(\mathbf{s}_{n} \cdot \mathbf{s}_{n+1}\right)+\mathbf{D}_{n} \cdot\left[\mathbf{s}_{n} \times \mathbf{s}_{n+1}\right]\right) .
$$

Here $\Omega_{n}$ is an external field at site $n$, and $J_{n}$ and $\mathbf{D}_{n}$ are the isotropic exchange interaction and the antisymmetric anisotropic exchange interaction or DM interaction between the neighbouring sites $n$ and $n+1$, respectively.

Extending the spin coordinate transformation used in Ref. [21] for models with nonuniform $J_{n}$ and $\mathbf{D}_{n}$ first we show how the terms with cross product can be eliminated from Hamiltonian (1) in special cases. If $\mathbf{D}_{n}$ has only one nonzero component $D_{n}^{z}$ one may perform local rotations about the $z$ axis introducing new spin operators $s_{n}^{x \prime}=s_{n}^{x} \cos \phi_{n}+s_{n}^{y} \sin \phi_{n}, s_{n}^{y^{\prime}}=-s_{n}^{x} \sin \phi_{n}+s_{n}^{y} \cos \phi_{n}, \phi_{n}=\varphi_{1}+\ldots+\varphi_{n-1}, \tan \varphi_{m}=\frac{D_{m}^{z}}{J_{m}}$ in terms of which (11) becomes 22 .

$$
H=\sum_{n} \Omega_{n} s_{n}^{z \prime}+\sum_{n}\left(\sqrt{J_{n}^{2}+D_{n}^{z^{2}}}\left(s_{n}^{x \prime} s_{n+1}^{x}{ }^{\prime}+s_{n}^{y^{\prime}} s_{n+1}^{y}{ }^{\prime}\right)+J_{n} s_{n}^{z^{\prime}} s_{n+1}^{z}{ }^{\prime}\right) .
$$

Note, that such transformation can be also applied to the transverse $X X$ chain in which $z$ spin components do not interact. Assume further that $\mathbf{D}_{n}$ has only one nonzero component $D_{n}^{x}$. Then the local rotations must be performed about the $x$ axis, i.e. $s_{n}^{y^{\prime}}=s_{n}^{y} \cos \phi_{n}+s_{n}^{z} \sin \phi_{n}, s_{n}^{z^{\prime}}=-s_{n}^{y} \sin \phi_{n}+s_{n}^{z} \cos \phi_{n}$ with $\tan \varphi_{m}=\frac{D_{m}^{x}}{J_{m}}$ resulting in

$$
H=\sum_{n} \Omega_{n}\left(\sin \phi_{n} s_{n}^{y^{\prime}}+\cos \phi_{n} s_{n}^{z \prime}\right)+\sum_{n}\left(J_{n} s_{n}^{x \prime} s_{n+1}^{x}{ }^{\prime}+\sqrt{J_{n}^{2}+D_{n}^{x^{2}}}\left(s_{n}^{y^{\prime}} s_{n+1}^{y}{ }^{\prime}+s_{n}^{z \prime} s_{n+1}^{z}{ }^{\prime}\right)\right) .
$$

Acting similarly for $\mathbf{D}_{n}=\left(0, D_{n}^{y}, 0\right)$ one finds that the transformed Hamiltonian is given by (3) with the replacement $D_{n}^{x} \rightarrow D_{n}^{y}, s^{x \prime} \rightarrow s^{y^{\prime}}, s^{y^{\prime}} \rightarrow-s^{x \prime}$. In the case when $\mathbf{D}_{n}$ has more than one nonzero components the described elimination can be performed if the orientation of $\mathbf{D}_{n}$ (but not necessarily its value $D_{n}=\sqrt{D_{n}^{x^{2}}+D_{n}^{y 2}+D_{n}^{z^{2}}}$ ) is site independent, i.e. $\frac{D_{n}^{x}}{D_{n}}, \frac{D_{n}^{y}}{D_{n}}, \frac{D_{n}^{z}}{D_{n}}$ do not depend on $n$. (Obviously, the particular site independent orientations of $\mathbf{D}_{n}$ reproduce the cases discussed above.) Really, in such a case we start from the global transformation of coordinate system with the Eulerian angles $\phi, \theta, \psi$ putting $\tan \phi=\frac{D_{n}^{y}}{D_{n}^{x}}, \theta=\frac{\pi}{2}$, $\tan \psi=\frac{D_{n}^{z}}{\sqrt{D_{n}^{x 2}+D_{n}^{y 2}}}$ and then perform the mentioned above local rotations about the $x$ axis with $\tan \varphi_{m}=\frac{D_{m}}{J_{m}}$ 
finding as a result

$$
\begin{aligned}
H= & \sum_{n} \Omega_{n}\left(\frac{D_{n}^{z}}{D_{n}} s_{n}^{x \prime}+\frac{\sqrt{D_{n}^{x^{2}}+D_{n}^{y^{2}}}}{D_{n}}\left(\cos \phi_{n} s_{n}^{y^{\prime}}-\sin \phi_{n} s_{n}^{z^{\prime}}\right)\right) \\
& +\sum_{n}\left(J_{n} s_{n}^{x \prime} s_{n+1}^{x}{ }^{\prime}+\sqrt{J_{n}^{2}+D_{n}^{2}}\left(s_{n}^{y^{\prime}} s_{n+1}^{y}{ }^{\prime}+s_{n}^{z^{\prime}} s_{n+1}^{z}{ }^{\prime}\right)\right) .
\end{aligned}
$$

It should be noted that the described transformations were applied to open chains. For cyclic chains they yield the presented expressions (2) - (ब1) at least up to the boundary term. Evidently, the thermodynamic properties of the initial and transformed Hamiltonians are identical. Due to these transformations (Eqs. (2) - (4)) one is able to exploit the broad knowledge on anisotropic Heisenberg chains and $X X$ chains. In what follow we shall use the thermodynamic equivalence of the initial and transformed Hamiltonians both in the analytical treatment and numerical computations.

We proceed considering the transverse $X X$ chain with DM interaction having only $z$ component

$$
H=\sum_{n} \Omega_{n} s_{n}^{z}+\sum_{n} J_{n}\left(s_{n}^{x} s_{n+1}^{x}+s_{n}^{y} s_{n+1}^{y}\right)+\sum_{n} D_{n}^{z}\left(s_{n}^{x} s_{n+1}^{y}-s_{n}^{y} s_{n+1}^{x}\right) .
$$

For the Hamiltonian (5) we are able to perform rigorous analytical calculations since after the Jordan-Wigner transformation it reduces to noninteracting spinless fermions. As it has been already mentioned thermodynamics of the model given by (5) is the same as of the transverse $X X$ chain (without DM interaction) with exchange

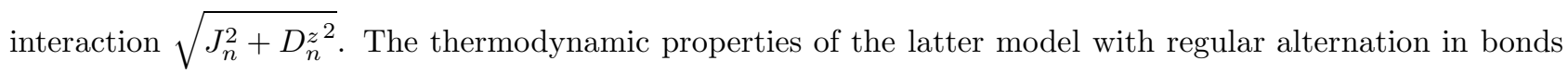
and fields having finite period $p$ have been examined recently with the help of continued fractions [20]. To study the spin-Peierls dimerization in the adiabatic limit we need the ground state energy of a spin chain with period $p=2$, i.e. with the sequence of parameters $\Omega_{1} J_{1} D_{1}^{z} \Omega_{2} J_{2} D_{2}^{z} \Omega_{1} J_{1} D_{1}^{z} \Omega_{2} J_{2} D_{2}^{z} \ldots$ Moreover, we assume the following reasonable dimerization ansatz $J_{1}=J(1+\delta), J_{2}=J(1-\delta), D_{1}^{z}=D^{z}(1+k \delta), D_{2}^{z}=D^{z}(1-k \delta)$, where $0 \leq \delta \leq 1$ is the dimerization parameter. It is argued that the directions of $\mathbf{D}$-vectors are not changed by the dimerization 10, 23. From Ref. [7] we know that the dependence on the intersite distance of the isotropic exchange interaction and DM interaction may be different. This effect is described by the parameter $k$. Putting $k=0$ one has a chain in which $D^{z}$ does not depend on the lattice distortion, whereas for $k=1$ the dependence of $D^{z}$ on the lattice distortion is as that for the isotropic exchange interaction $J$. The latter case with $\Omega_{n}=0$ was considered in [16]. Besides, we bear in mind that, as a rule, the value of DM interaction is significantly smaller than the value of isotropic exchange interaction [7].

Further, we consider the case of zero temperature and look for the total energy per site $\mathcal{E}(\delta)$ which consists of the magnetic part $e_{0}(\delta)$ and the elastic part $\alpha \delta^{2}, \alpha>0$. From Ref. 20 we know the exact expression for the magnetic ground state energy

$$
e_{0}(\delta)=-\frac{1}{\pi} \mathrm{b}_{1} \mathrm{E}\left(\psi, \frac{\mathrm{b}_{1}^{2}-\mathrm{b}_{2}^{2}}{\mathrm{~b}_{1}^{2}}\right)-\frac{1}{2}\left|\Omega_{1}+\Omega_{2}\right|\left(\frac{1}{2}-\frac{\psi}{\pi}\right)
$$




$$
\begin{array}{r}
\mathrm{b}_{1,2}=\frac{1}{2} \sqrt{\left(\Omega_{1}-\Omega_{2}\right)^{2}+\left(I_{1} \pm I_{2}\right)^{2}}, \\
I_{1,2}=\sqrt{J^{2}(1 \pm \delta)^{2}+D^{z^{2}}(1 \pm k \delta)^{2}}, \\
\psi= \begin{cases}0, & \text { if } \mathrm{b}_{1} \leq \frac{1}{2}\left|\Omega_{1}+\Omega_{2}\right|, \\
\arcsin \sqrt{\frac{\mathrm{b}_{1}^{2}-\frac{1}{4}\left(\Omega_{1}+\Omega_{2}\right)^{2}}{\mathrm{~b}_{1}^{2}-\mathrm{b}_{2}^{2}},}, & \text { if } \quad \mathrm{b}_{2} \leq \frac{1}{2}\left|\Omega_{1}+\Omega_{2}\right|<\mathrm{b}_{1}, \\
\frac{\pi}{2}, & \text { if } \frac{1}{2}\left|\Omega_{1}+\Omega_{2}\right|<\mathrm{b}_{2},\end{cases}
\end{array}
$$

where $\mathrm{E}\left(\psi, a^{2}\right) \equiv \int_{0}^{\psi} d \phi \sqrt{1-a^{2} \sin ^{2} \phi}$ is the elliptic integral of the second kind. We also seek for a nonzero solution $\delta^{\star} \neq 0$ of the equation $\frac{\partial \mathcal{E}(\delta)}{\partial \delta}=0$ that can be easily derived from (6). In what follows we consider the case of a uniform transverse field $\Omega_{1}=\Omega_{2}=\Omega_{0} \geq 0$. In the limit $\delta \ll 1$ valid for hard lattices (having large values of $\alpha$ and corresponding to the experimental situation) one finds $\mathrm{b}_{1}=I, \mathrm{~b}_{2}=I \aleph \delta$ with $I=\sqrt{J^{2}+D^{z^{2}}}$ and $\aleph=\frac{J^{2}+k D^{z 2}}{J^{2}+D^{22}}$. Instead of Eq. (6) one then has

$$
\begin{gathered}
e_{0}(\delta)=-\frac{I}{\pi} \mathrm{E}\left(\psi, 1-\aleph^{2} \delta^{2}\right)-\Omega_{0}\left(\frac{1}{2}-\frac{\psi}{\pi}\right), \\
\psi= \begin{cases}0, & \text { if } I<\Omega_{0}, \\
\arcsin \sqrt{\frac{I^{2}-\Omega_{0}^{2}}{I^{2}\left(1-\aleph^{2} \delta^{2}\right)},} & \text { if } I \aleph \delta \leq \Omega_{0}<I, \\
\frac{\pi}{2}, & \text { if } \quad \Omega_{0}<I \aleph \delta,\end{cases}
\end{gathered}
$$

whereas the equation for $\delta^{\star}$ reads

$$
\frac{2 \pi \alpha}{I}=\frac{\aleph^{2}}{1-\aleph^{2} \delta^{\star^{2}}}\left(F\left(\psi, 1-\aleph^{2} \delta^{\star^{2}}\right)-E\left(\psi, 1-\aleph^{2} \delta^{\star 2}\right)\right),
$$

where $\mathrm{F}\left(\psi, a^{2}\right) \equiv \int_{0}^{\psi} \frac{d \phi}{\sqrt{1-a^{2} \sin ^{2} \phi}}$ is the elliptic integral of the first kind.

Consider at first the case $\Omega_{0}=0$. After rescaling $I \rightarrow J, \frac{\alpha}{\aleph^{2}} \rightarrow \alpha, \aleph \delta^{\star} \rightarrow \delta^{\star}$ one finds that Eq. (8) is exactly the same as considered in Ref. [3] and thus $\delta^{\star} \sim \frac{1}{\aleph} \exp \left(-\frac{2 \pi \alpha}{I \aleph^{2}}\right)$. Thus for $k=1(\aleph=1)$ nonzero $D^{z}$ leads to an increasing of the dimerization parameter $\delta^{\star}$, whereas for $k=0(\aleph \leq 1)$ nonzero $D^{z}$ leads to a decreasing of $\delta^{\star}$. Let us pass to the case $0<\Omega_{0}<I$. Varying $\delta^{\star}$ in the r.h.s. of Eq. (8) from 0 to 1 one calculates a lattice parameter $\alpha$ for which the taken value of $\delta^{\star}$ realizes an extremum of $\mathcal{E}(\delta)(\mathbb{T})$. One immediately observes that for $\frac{\Omega_{0}}{I \aleph} \leq \delta^{\star}$ the dependence $\alpha$ versus $\delta^{\star}$ remains as that in the absence of the field, whereas for $0 \leq \delta^{\star}<\frac{\Omega_{0}}{I \aleph}$ the calculated quantity $\alpha$ starts to decrease. From this one concludes that the field $\frac{\Omega_{0}}{I}=\exp \left(-\frac{2 \pi \alpha}{I \aleph^{2}}\right)$ makes the dimerization unstable against the uniform phase. The latter relation tells us that nonzero $D^{z}$ increases the value of that field for $k=1$ and decreases it for $k=0$. It is known [2] that the increasing of the external field leads to a transition from the dimerized phase to the incommensurate phase rather than to the uniform phase. However, the former phase cannot appear within the frames of the adopted ansatz for the lattice distortions $\delta_{1} \delta_{2} \delta_{1} \delta_{2} \ldots, \delta_{1}+\delta_{2}=0$.

After the discussion of the limit $\delta \ll 1$ we now present the results for arbitrary $0 \leq \delta \leq 1$ based on (6). In Figs. 1,2 we plot the changes of the total energy $\mathcal{E}(\delta)-\mathcal{E}(0)(6)$ vs $\delta$ and the nonzero solution $\delta^{\star}$ of equation 
$\frac{\partial \mathcal{E}(\delta)}{\partial \delta}=0$ vs $\alpha$, respectively, for various strengths of DM interaction. These results confirm that for $k=1$ DM interaction $D^{z}$ acts in favour of dimerization, whereas for $k=0$ against it. To understand the validity of the data obtained by exact diagonalization of finite chains that will be used below for the Heisenberg chain we present in Fig. 1 also the numerical results $\mathcal{E}(\delta)-\mathcal{E}(0)$ vs $\delta$ for $N=24$ spins (open boundary conditions). Note, that although the finite chain results for $N=24$ still overestimate noticeably the value of $\delta^{\star}$ and the depth $\mathcal{E}\left(\delta^{\star}\right)-\mathcal{E}(0)$, however, and this is most important, they reproduce qualitatively correctly the influence of $D^{z}$ in both cases $k=1$ and $k=0$. The possible influences of $D^{z}$ are reproduced correctly even for shorter chains of $N=16,20$ spins. Moreover, with increasing the chain length from $N=16$ to 24 the numerical data approach the analytical ones valid for $N \rightarrow \infty$. This expected tendency can be also seen by comparison curves 1 for $N=24, N=28$ (the computation in this case becomes already very time consuming) and $N \rightarrow \infty$ in Fig. 1.

Let us turn to the Heisenberg chain with DM interaction. Assume that the vectors $\mathbf{D}_{n}$ have the same orientation at all sites, e.g. in the $z$ direction. (The assumption $\mathbf{D}_{n}=\left(0,0, D_{n}^{z}\right)$ does not lead to a loss of generality if $\Omega_{0}=0$.) In such case the influence of DM interaction on thermodynamics follows from a study of thermodynamic properties of the Heisenberg chain with anisotropic exchange interaction (2). We immediately find the appearance of $X X$ anisotropy, since the interaction between $x$ and $y$ spin components becomes $\sqrt{J^{2}(1 \pm \delta)^{2}+D^{2}(1 \pm k \delta)^{2}}\left(\approx \sqrt{J^{2}+D^{2}}(1 \pm \aleph \delta)\right.$ for $\left.\delta \ll 1\right)$ whereas between $z$ spin components it remains $J(1 \pm \delta)$. Restricting ourselves to small $\delta$ we perform the redefinitions $\sqrt{J^{2}+D^{2}}=J^{\prime}, \aleph \delta=\delta^{\prime}, \frac{\alpha}{\aleph^{2}}=\alpha^{\prime}$. As a result we come to the anisotropic alternating Heisenberg chain with $x x$ and $y y$ interactions $J^{\prime}\left(1 \pm \delta^{\prime}\right)$ and $z z$ interaction $J^{\prime} \Delta\left(1 \pm \frac{\delta^{\prime}}{\aleph}\right), \Delta=\frac{J}{\sqrt{J^{2}+D^{2}}}$ and the expression for the elastic energy per site $\alpha^{\prime} \delta^{\prime 2}$. Any increase of $D$ results in increasing of intersite coupling $J^{\prime}$ and increasing of $X X$ anisotropy manifested by going of $\Delta$ from $1(D=0)$ to $0(D \rightarrow \infty)$. Hence, the Heisenberg chain should start to exhibit a behaviour inherent in the $X X$ chain while $D$ becomes large. Omitting the role of a change in the $z z$ interaction, which becomes less important while $D$ increases, and bearing in mind the corresponding analysis for the $X X$ chain based on (8), we may expect that for $\Omega_{0}=0$ the dimerized phase will be enhanced for $k=1$ (since $\alpha^{\prime}=\alpha$ ) and will be suppressed for $k=0$ (since $\alpha^{\prime}>\alpha$ ). The numerical results for $\mathcal{E}(\delta)-\mathcal{E}(0)$ vs $\delta$ for the Heisenberg chain of $N=24$ sites (open boundary conditions) shown in Fig. 3 confirm this expectation. As can be seen from the displayed plots the general tendency for the changes caused by DM interaction is the same for $X X$ and Heisenberg chains.

To conclude, we have examined the stability of some spin- $\frac{1}{2}$ transverse $X X$ and Heisenberg chains with respect to dimerization in the presence of DM interaction analysing the dependence of the ground state energy on dimerization parameter. If the orientations of $\mathbf{D}_{n}$ are the same at all sites essential simplification occurs, i.e. the terms with cross product can be eliminated from the Hamiltonian resulting in the appearance of the anisotropy in the exchange interaction. In addition, the external field becomes more complicated having three 
site dependent components (see (田)). The transformed Hamiltonian may be more transparent and convenient for further analytical or numerical treatment. For the transverse $X X$ chain we have found that DM interaction having only $z$ component may act both in favour of the dimerization or against it. The result of its influence depends on the dependence of DM interaction on the amplitude of lattice distortion in comparison with a corresponding dependence of the isotropic exchange interaction. For the Heisenberg chain DM interaction having the same orientation at all sites leads to $X X$ anisotropy (and extra nonuniform on-site fields in the presence of an external field). For the Heisenberg chain without field we have observed qualitatively the same behaviour as in the $X X$ chain determined by the dependence of DM interaction on the lattice distortion.

O. D. and J. R. acknowledge the kind hospitality of the Max Planck Institute for the Physics of Complex Systems, Dresden in the end of 1999 when a main part of the paper was done. The present study was partly supported by the DFG (projects 436 UKR $17 / 20 / 98$ and Ri $615 / 6-1$ ).

\section{References}

[1] M. Hase, I. Terasaki, and K. Uchinokura, Phys. Rev. Lett. 70, 3651 (1993).

[2] For a review see: J. P. Boucher and L. P. Regnault, J. Phys. I France 6, 1939 (1996).

[3] P. Pincus, Solid State Commun. 9, 1971 (1971);

G. Beni and P. Pincus, J. Chem. Phys. 57, 3531 (1972).

[4] J. H. Taylor and G. Müller, Physica A 130, 1 (1985) (and references therein).

[5] K. Okamoto and K. Yasumura, J. Phys. Soc. Jpn. 59, 993 (1990) (and references therein);

K. Okamoto, J. Phys. Soc. Jpn. 59, 4286 (1990);

K. Okamoto, Solid State Commun. 83, 1039 (1992).

[6] K. Kawasaki, N. Maya, A. Kouzuki, and K. Nakamura, J. Phys. Soc. Jpn. 66, 839 (1997).

[7] T. Moriya, Phys. Rev. 120, 91 (1960).

[8] I. Yamada, M. Nishi, and J. Akimitsu, J. Phys.: Condens. Matter 8, 2625 (1996).

[9] V. N. Glazkov, A. I. Smirnov, O. A. Petrenko, D. Mㄷ․ Paul, A. G. Vetkin, and R. M. Eremina, J. Phys.: Condens. Matter 10, 7879 (1998).

[10] H. Nojiri, H. Ohta, S. Okubo, O. Fujita, J. Akimitsu, and M. Motokawa, J. Phys. Soc. Jpn. 68, 3417 (1999). 
[11] D. Coffey, T. M. Rice, and F. C. Zhang, Phys. Rev. B 44, 10112 (1991).

[12] L. Shekhtman, O. Entin-Wohlman, and A. Aharony, Phys. Rev. Lett. 69, 836 (1992).

[13] Q. Xia and P. S. Riseborough, J. Appl. Phys. 67, 5478 (1990).

[14] W. Koshibae, Y. Ohta, and S. Maekawa, Physica C 185-189, 1509 (1991).

[15] A. Voigt and J. Richter, J. Phys.: Condens. Matter 8, 5059 (1996).

[16] A. A. Zvyagin, Zh. Eksp. Teor. Fiz. 98, 1396 (1990) (in Russian);

A. A. Zvyagin, Phys. Lett. A 158, 333 (1991).

[17] I. Yamada, H. Fujii, and M. Hidaka, J. Phys.: Condens. Matter 1, 3397 (1989);

T. Ishii and I. Yamada, J. Phys.: Condens. Matter 2, 5771 (1990);

I. Tsukada, J. Takeya, T. Masuda, and K. Uchinokura, preprint cond-mat/0004403.

[18] A. S. T. Pires and M. E. Gouvêa, J. Magn. Magn. Mater. 212, 251 (2000) (and references therein).

[19] J. N. McElearney and S. Merchant, Phys. Rev. B 18, 3612 (1978).

[20] O. Derzhko, J. Richter, and O. Zaburannyi, Phys. Lett. A 262, 217 (1999);

O. Derzhko, J. Richter, and O. Zaburannyi, cond-mat/0001014.

[21] M. Oshikawa and I. Affleck, Phys. Rev. Lett. 79, 2883 (1997).

[22] After this work was completed (preprint mpi-pks/9912013 (http://www.mpipks-dresden.mpg.de) a preprint by D. N. Aristov and S. V. Maleyev (preprint cond-mat/0004272 (http://xxx.lanl.gov)) appeared, in which a similar transformation was used while studying the influence of the DM interaction in the Heisenberg chain for the observables in the polarized neutron scattering experiments.

[23] Note, however, that according to Refs. 8, 10] $\mathbf{D}$-vectors in $\mathrm{CuGeO}_{3}$ are parallel to one another along the $\mathbf{a}$-axis and they are alternated along the $\mathbf{b}$-axis.

\section{FIGURE CAPTURES}

FIGURE 1. Dependence $\mathcal{E}(\delta)-\mathcal{E}(0)$ (6) vs $\delta$ for the transverse $X X$ chain with DM interaction. $J=2$, $\Omega_{0}=0, \alpha=0.8, D^{z}=0$ (curve 1), $D^{z}=0.4,0.8$ for $k=1$ (curves 2 and 3, respectively) and $k=0$ (curves 4 and 5 , respectively). Solid curves correspond to analytical calculations $(N \rightarrow \infty)$, whereas dotted ones to exact diagonalization results $(N=24)$. The dashed curve corresponds to the numerical results for $N=28$. 
FIGURE 2. Dependence $\delta^{\star}$ vs $\alpha$ for the transverse $X X$ chain with DM interaction. $J=2, \alpha=0.8, \Omega_{0}=0$ (a), $\Omega_{0}=0.2(\mathrm{~b}), D^{z}=0$ (curve 1), $D^{z}=0.4,0.8$ for $k=1$ (curves 2 and 3 , respectively) and $k=0$ (curves 4 and 5 , respectively).

FIGURE 3. Dependence $\mathcal{E}(\delta)-\mathcal{E}(0)$ vs $\delta$ for the Heisenberg chain with DM interaction obtained by exact diagonalization of finite chains. $J=2, \Omega_{0}=0, \alpha=0.8, D=0$ (curve 1), $D=0.4,0.8$ for $k=1$ (curves 2 and 3 , respectively) and $k=0$ (curves 4 and 5 , respectively). We also plotted the dependence $\mathcal{E}(\delta)-\mathcal{E}(0)$ vs $\delta$ for the corresponding $X X$ chain with $D=0$ (i.e. curves 1 in Fig. 1) as it follows from analytical calculation for $N \rightarrow \infty$ (dashed curve) and numerical computation for $N=24$ (dotted curve). 


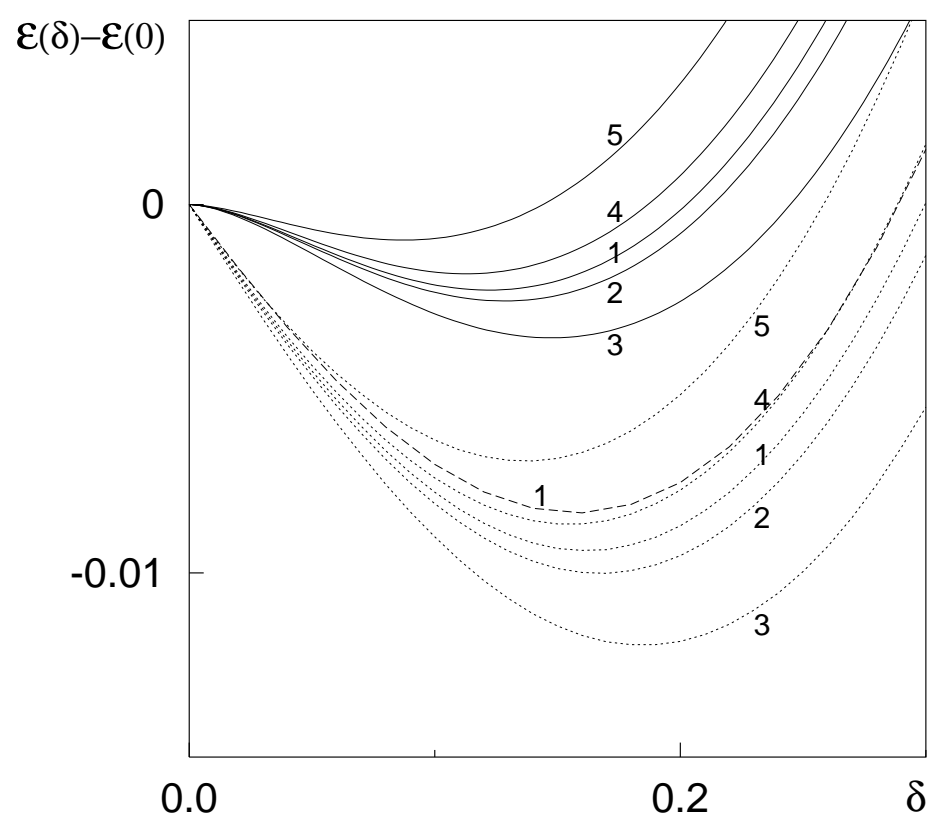

Figure 1: Dependence $\mathcal{E}(\delta)-\mathcal{E}(0)$ (6) vs $\delta$ for the transverse $X X$ chain with DM interaction. $J=2, \Omega_{0}=0, \alpha=0.8$, $D^{z}=0$ (curve 1), $D^{z}=0.4,0.8$ for $k=1$ (curves 2 and 3, respectively) and $k=0$ (curves 4 and 5 , respectively). Solid curves correspond to analytical calculations $(N \rightarrow \infty)$, whereas dotted ones to exact diagonalization results $(N=24)$. The dashed curve corresponds to the numerical results for $N=28$. 


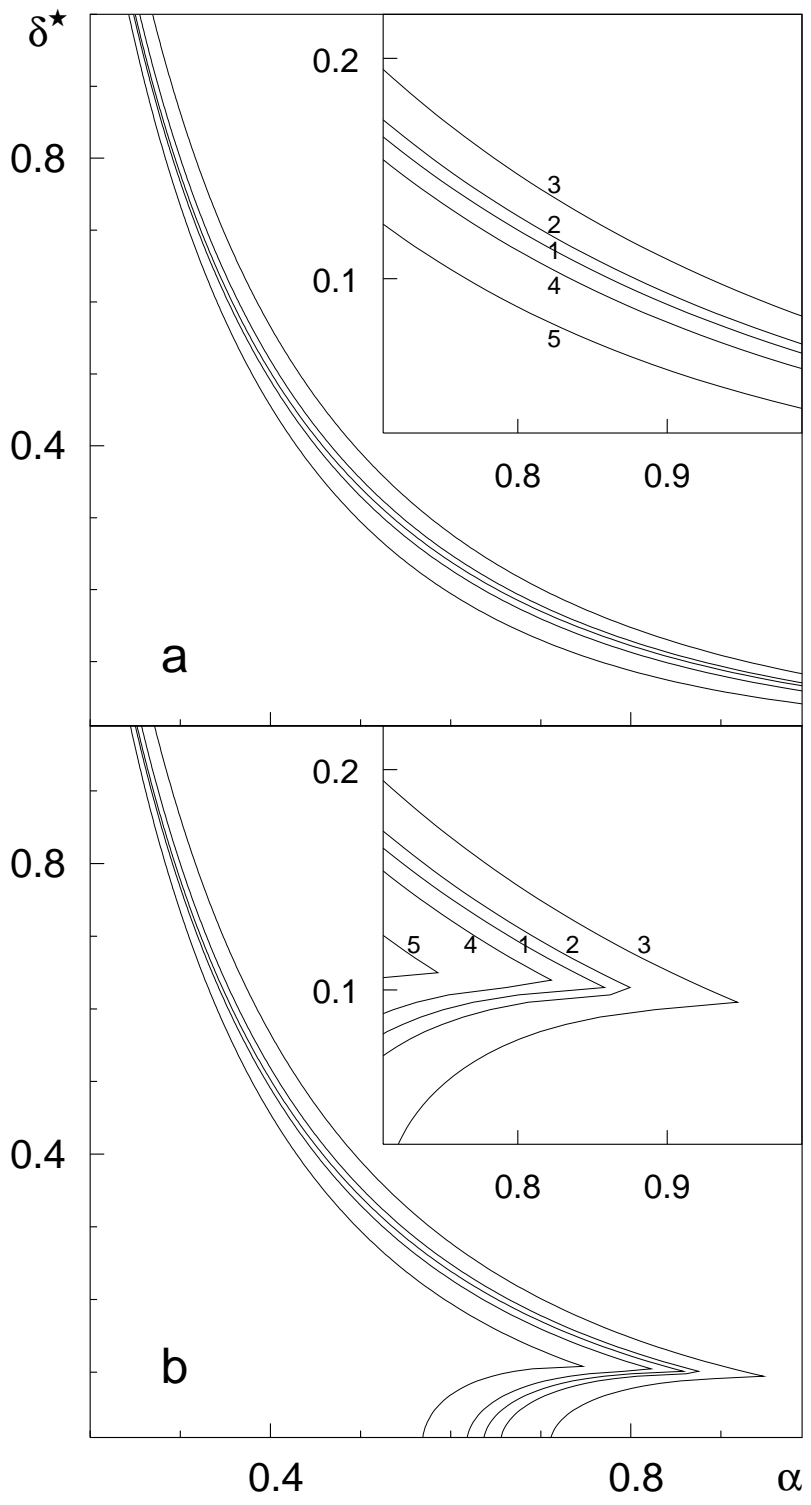

Figure 2: Dependence $\delta^{\star}$ vs $\alpha$ for the transverse $X X$ chain with DM interaction. $J=2, \alpha=0.8, \Omega_{0}=0$ (a), $\Omega_{0}=0.2$ (b), $D^{z}=0$ (curve 1), $D^{z}=0.4,0.8$ for $k=1$ (curves 2 and 3 , respectively) and $k=0$ (curves 4 and 5 , respectively). 


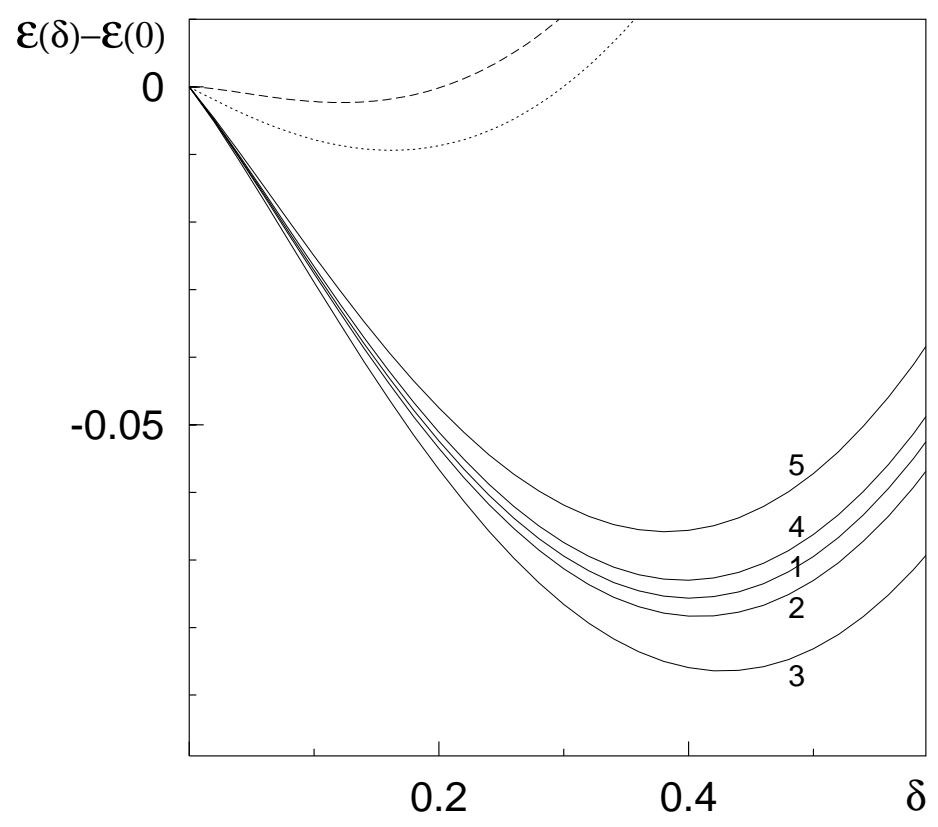

Figure 3: Dependence $\mathcal{E}(\delta)-\mathcal{E}(0)$ vs $\delta$ for the Heisenberg chain with DM interaction obtained by exact diagonalization of finite chains. $J=2, \Omega_{0}=0, \alpha=0.8, D=0$ (curve 1), $D=0.4,0.8$ for $k=1$ (curves 2 and 3 , respectively) and $k=0$ (curves 4 and 5 , respectively). We also plotted the dependence $\mathcal{E}(\delta)-\mathcal{E}(0)$ vs $\delta$ for the corresponding $X X$ chain with $D=0$ (i.e. curves 1 in Fig. 1) as it follows from analytical calculation for $N \rightarrow \infty$ (dashed curve) and numerical computation for $N=24$ (dotted curve). 